\title{
Ear-EEG: Continuous Brain Monitoring
}

\author{
David Looney, ${ }^{1}$ Preben Kidmose, ${ }^{2}$ Mary J. Morrell, ${ }^{1,3}$ and Danilo P. Mandic ${ }^{1}$
}

${ }^{1}$ Imperial College London, UK; ${ }^{2}$ Aarhus University, Denmark; ${ }^{3}$ Sleep Unit, Royal Brompton Hospital, London, UK david.looney06@imperial.ac.uk, pki@iha.dk,.m.morrell@imperial.ac.uk, d.mandic@imperial.ac.uk

\begin{abstract}
Project description I The aim of this submission has been twofold: (i) to present a radically new way of recording EEG comfortably and unobtrusively over long time-periods in natural environments. This break-through has been achieved using electrodes embedded on a customized earpiece as typically used in hearing aids (EarEEG); (ii) to illustrate the potential of Ear-EEG as an enabling technology for a number of uses beyond traditional $\mathrm{BCl}$ which are currently limited by inconvenience of standard EEG recording. We show that Ear-EEG enables both conventional $\mathrm{BCl}$ and nextgeneration applications such as the evaluation of hearing capability and the monitoring of fatigue and drowsiness.
\end{abstract}

\section{Requirements of Wearable EEG Technology}

Opportunities for EEG-based $\mathrm{BCl}$ are rapidly expanding beyond the traditional aim of high-performance communication pathways for paralyzed patients. The goal of these applications is continuous brain monitoring and measurement of brain state [1]; examples include the measuring drowsiness to prevent road accidents [1], modelling stress to optimize performance in the work place, monitoring sleep disorders [2] and the evaluation of sensory deficit. However widespread use of $\mathrm{BCl}$ is limited by conventional recording systems, which are bulky, cumbersome and primarily operate in the specialist setting.

This highlights the need for wearable systems which allow long-term recordings in natural environments. Such systems are particularly useful in applications where a trade-off in performance is acceptable to enhance user comfort. Improvements in battery size and dry electrode technologies ${ }^{1}$ are advances, but they do not negate the need for on-scalp electrodes attached by cap, adhesives or headset, which inevitably make the recording process uncomfortable. In order for EEG-based $\mathrm{BCl}$ to be adopted more widely and in natural environments, the recording technology should be [3]:

- Discreet - not clearly visible or stigmatising,

- Unobtrusive - comfortable to wear and impeding the user as little as possible,

- User-friendly - users should be able to attach and operate the devices themselves.

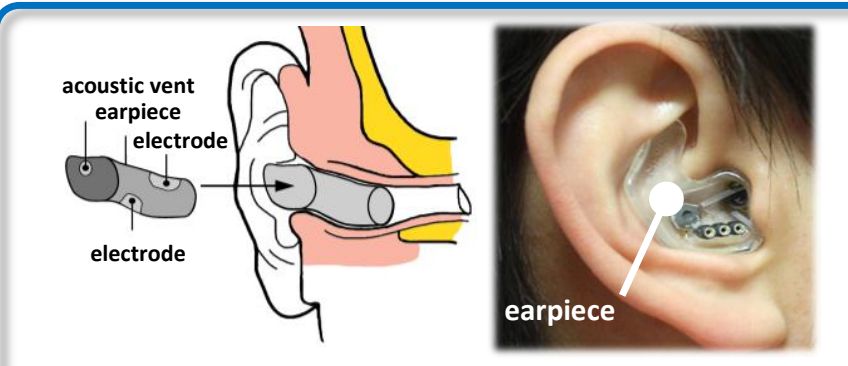

Fig. 1: [Left] A sketch of the Ear-EEG prototype and its location inside the ear. [Right] Photograph of earpiece worn in-ear

\footnotetext{
${ }^{1}$ Conventional electrodes require the use of conductive gel to enable an electrical connection between the electrodes and the scalp.
}

\section{The Ear-EEG Recording Concept}

We recently introduced Ear-EEG [3-6], a technology which satisfies core user requirements (unobtrusive, discreet and user-friendly). This represents a significant step forward in wearable EEG whereby, benefiting from the underlying hearing aid platform, all electrodes (including reference and ground) are embedded on a customized earpiece placed within the ear canal and external ear (pinna), see Fig. 1. The tight fit between earpiece and ear canal ensures that electrodes are held firmly in place, thus overcoming some critical EEG obstacles - motion, electromagnetic, and ocular artifacts (see Fig 2 [upper]) and experiment repeatability.

The Ear-EEG approach has already been rigorously validated against standard (scalp) EEG in time and frequency signal characteristics for a range of responses [3-6]. Fig. 2 [upper] demonstrates the robustness of Ear-EEG to common sources of artifacts, while Fig. 2 [centre] illustrates similar alpha activity - a response underpinning attention and fatigue modeling - in Ear-EEG and scalp EEG within the timefrequency domain. The Ear-EEG responses closely match those obtained for scalp-EEG electrodes at the temporal region, where the primary auditory cortex is located. While signal amplitudes measured from within the ear are weaker, so too is the noise, so that for auditory responses (Fig. 2 [lower, left]) the signal-to-noise ratios (SNR) are similar [4]. Fig. 2 [lower right]) establishes a basis for the conventional communication-type $\mathrm{BCl}$ operation of Ear-EEG [6].

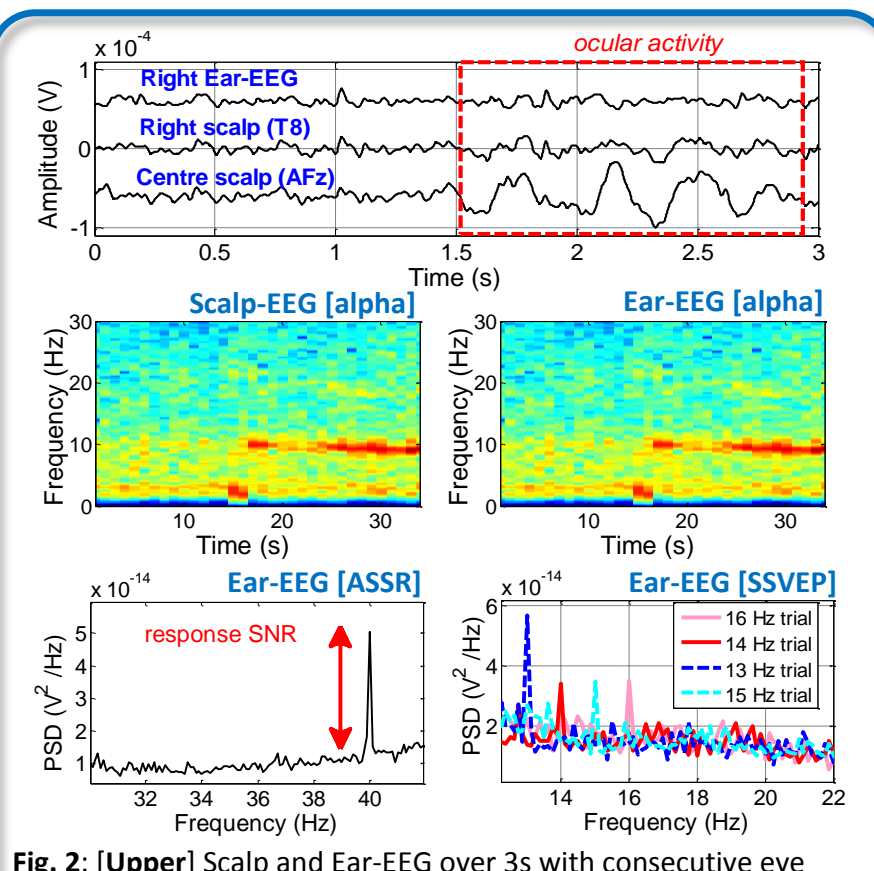

Fig. 2: [Upper] Scalp and Ear-EEG over 3s with consecutive eye blinking starting at 1.5s, Ear-EEG is less sensitive to ocular artifacts. [Centre] Time-frequency plots as subject closes eyes from 15-35s, with increased activity visible for scalp [left] and Ear-EEG [right] in the alpha range $(8-12 \mathrm{~Hz})$. [Lower, left] Auditory $(40 \mathrm{~Hz}$ amplitude modulated stimulus) and [Lower, right] visual $(13,14,15$ and $16 \mathrm{~Hz}$ stimuli) Ear-EEG steady state responses, with the latter illustrating traditional communication $\mathrm{BCl}$ (nominated for $2012 \mathrm{BCl}$ Award [6]) 


\section{Continuous Brain Monitoring: Case Studies}

The Ear-EEG concept offers an innovative breakthrough for recording EEG over long time periods. At present, the analysis is achieved via a simple prototype (see Fig. 1) but with on-going developments Ear-EEG will be a tiny battery powered brain monitoring device with gel-free electrodes that, like modern hearing aids, will perform both the recording and signal processing in situ. We have already established that Ear-EEG is capable of performing conventional communication $\mathrm{BCl}$. The next phase in $\mathrm{BCl}-$ continuous brain monitoring ${ }^{2}$ - is here illustrated via two case studies using Ear-EEG.

\section{Case Study 1: Hearing Threshold Estimation}

The World Health Organisation estimate that hearing impairment affects more than 250 million people worldwide, making it the most common sensory deficit. A significant retail expense in hearing devices - an industry with revenues in excess of $\$ 6$ billion/year - is the fitting of hearing aids, the basis of which is the hearing threshold level (HTL). Typically HTL is estimated via behavioural hearing tests at an audiology clinic; however in many cases hearing loss is progressive or fluctuating (Meniere's disease, auditory neuropathy) and requires continuous assessment.

The Ear-EEG platform accommodates a loudspeaker, as in hearing aids, inducing auditory evoked potentials relevant to HTL into the EEG. The amplitudes of the so-induced EEG responses reflect the intensities of the stimuli, enabling a model of HTL and continuous hearing aid adaptation to match progressive/fluctuating hearing loss without the need of an audiologist. Fig. 3 validates Ear-EEG HTL estimation for a well-established protocol based on the auditory steady state response (ASSR) [7]. It depicts a high level of similarity between the ASSR SNR (e.g. Fig. 2 [lower left]) recorded from a conventional scalp electrode located in the temporal region (Tp9) and an Ear-EEG electrode (ELB referenced to ELH, see [4]) for various stimulus sound pressure levels (SPL).
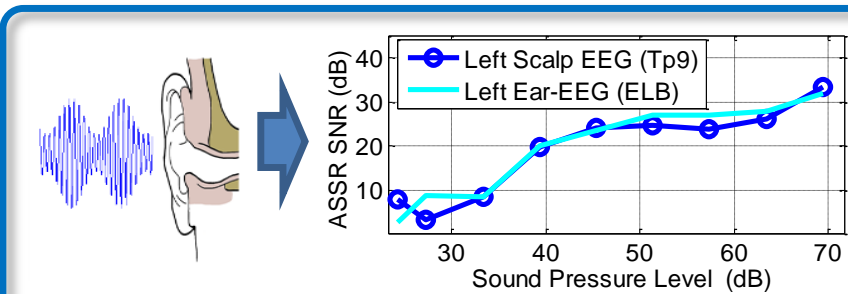

Fig. 3: Hearing Threshold Estimation. ASSR SNR, ratio in spectral domain of the response peak to background EEG (e.g. Fig. 2, lower left), for scalp and ear electrodes for stimuli of increasing SPLS

Case Study 2: Fatigue Estimation

Many occupations and daily activities require prolonged periods of vigilance. In the transport industry a lapse in concentration can be fatal. The Royal Society for the Prevention of Accidents, estimate that driver fatigue accounts for $20 \%$ of road accidents in Great Britain. Yet there is still no readily available device that can objectively and reliably detect a loss of sustained attention.

\footnotetext{
${ }^{2}$ To ensure a fair comparison between scalp and Ear-electrodes, EEG was recorded for both approaches using the same amplifier (g.USBamp by g.tec) which has several independent blocks of inputs. On-scalp reference and ground electrodes were placed at, respectively, chin and $\mathrm{Cz}$ (study 1 ) and earlobe and Fpz (study 2) based on the 10-20 system. All ear-electrodes were inside the ear including reference and ground (see [4] for details).
}

Fig. 2 [centre, left] shows that Ear-EEG can track alpha activity with high accuracy. As increases in alpha power are also caused by drowsiness we next demostrate how Ear-EEG models drowsiness on par with scalp: highlighting its future role in maintaining vigliance (e.g. via a loudspeaker alert). Our study was based on the Oxford Sleep Resistance Test; a functional test of attention and drowsiness [8], whereby a subject was instructed to press a button in response to periodic visual stimuli. Missed consecutive stimuli were considered indicators of attention lapses caused by fatigue induced by requesting the subject to reduce their sleeping hours before the test. The results of the response test, and the corresponding level of alpha power estimated using scalp- and ear- electrodes are shown in Fig. 4. Observe that consecutive error events are accompanied by clear increases in alpha power in both sets of electrodes which exhibit a high level of similarity.

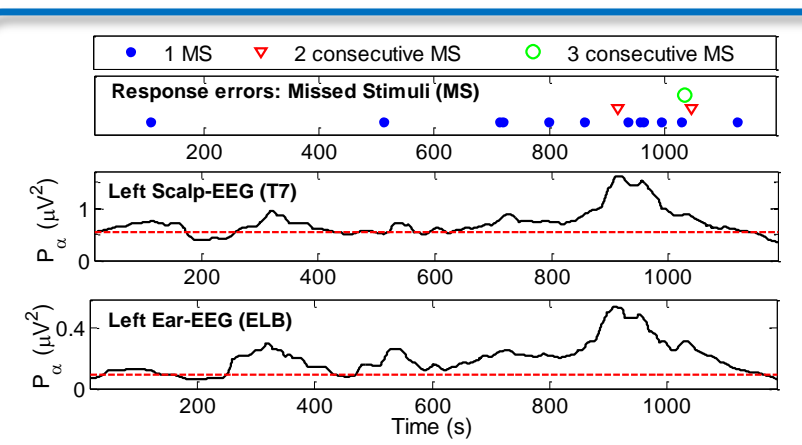

Fig. 4: Fatigue Estimation. [Upper] Response errors: missed stimuli (MS) events, single and consecutive. [Centre \& Lower] Alpha power (solid black line) for the scalp and ear electrodes respectively. For comparison, the subject's average alpha power estimated during a non-fatigue recording is also given (dashed red line)

\section{Summary}

Ear-EEG is a breakthrough in wearable sensing that has the potential to be used in non-specialist environment over long time periods. It is robust, discreet and comfortable. We here highlight the usefulness of Ear-EEG for the next stage of $\mathrm{BCl}-$ continuous brain monitoring - in two case studies considering estimation of hearing threshold and fatigue, with all electrodes, including reference and ground, embedded on the earpiece. This ope

\section{REFERENCES}

[1] B. Blankertz et al., "The Berlin brain-computer interface: Non-medical uses of BCI technology," Frontiers in Neuroscience, vol. 4, no. 198, 2010.

[2] N. R. Ward, M. R. Cowie, S. D. Rosen, V. Roldao, M. De Villa M, T. A McDonagh, A. Simonds, and M. J. Morrell, "Utility of overnight pulse oximetry and heart rate variability analysis to screen for sleep-disordered breathing in chronic heart failure", Thorax, vol. 67, no. 11, pp. 1000-1005, 2012.

[3] D. Looney, P. Kidmose, C. Park, M. Ungstrup, M. L. Rank, K. Rosenkranz, and D. P. Mandic, "The in-the-ear recording concept", IEEE Pulse Magazine, vol. 3, no. 6, pp. 32-42, 2012.

[4] P. Kidmose, D. Looney, and D. P. Mandic, "Auditory evoked responses from Ear-EEG recordings", in Proc. of the Int. Conference of the IEEE Engineering in Medicine and Biology Society (EMBC), 2012, pp.586-589.

[5] P. Kidmose, D. Looney, M. Ungstrup, M. L. Rank, and D. P. Mandic, "Evoked potentials from Ear-EEG," IEEE Transactions on Biomedical Engineering (accepted), 2013

[6] D. Looney, P. Kidmose, and D. P. Mandic, "Ear-EEG: User-centered, wearable \& 24/7 BCI", nominated for the Annual BCI Research Award, 2012

[7] B. Cone-Wesson, R. C. Dowell, D. Tomlin, G. Rance, and W. J. Ming, "The auditory steady-state response: Comparisons with the auditory brainstem response" Journal of the American Academy of Audiology, vol. 13, no. 4, pp. 173 187, 2002.

[8] R. J. Davies, L. S. Bennett, J. R. Stradling, "What is an arousal and how should it be quantified?", Sleep Medicine Reviews, vol. 1, no. 2, pp. 87-95, 1997. 